\title{
Thermal dependence of magnetocrystalline anisotropy and magnetostriction constants of single crystal $\mathrm{Fe}_{2,4} \mathbf{T i}_{\mathbf{0 . 6 1}} \mathbf{O}_{\mathbf{4}}$
}

\author{
Sanghamitra Sahu and Bruce M. Moskowitz \\ Department of Geology and Geophysics and Institute for Rock Magnetism, University of Minnesota \\ Minneapolis, Minnesota
}

\begin{abstract}
The temperature dependence of the magnetocrystalline anisotropy $\left(\mathbf{K}^{\prime}\right)$ and magnetostriction $(\lambda)$ constants of single crystal $\mathrm{Fe}_{2.4} \mathrm{Ti}_{0.61} \mathrm{O}_{4}$ (TM61) is measured using torque magnetometry and strain gage techniques in the temperature range between $300 \mathrm{~K}$ and $T_{c}$. At all temperatures, $K^{\prime}$ is positive making the $\langle 100\rangle$ instead of $\langle 111\rangle$ crystal directions the easy axes of magnetization in TM61. The thermal dependence of $K^{\prime}(T)$ varies as $\left(1-T / T_{c}\right)^{2.2}$. In terms of the saturation magnetization, $K^{\prime}(T) \propto M_{2}(T)$ " where $n=6$, a much weaker dependence than that found for magnetite $(n=8-10)$. We also observed that $K^{\prime} \propto \lambda^{1-2}$, consistent with $K^{\prime} \propto M_{1}^{6}$. These results show that the magnetoelastic contribution to the magnetocrystalline anisotropy dominates the anisotropy constant of TM61 at elevated temperature and that the effects of stress cannot be neglected in theories of thermoremanence and micromagnetic models for intermediate titanomagnetites commonly found in oceanic basalts.
\end{abstract}

\section{Introduction}

High temperature behavior of material properties like magnetocrystalline anisotropy $\left(K_{1}\right)$ and magnetostriction $(\lambda)$ play key roles in the acquisition of natural remanent magnetization and its long term stability. The thermal behavior of these constants, along with the magnetic exchange constant and saturation magnetization $\left(M_{2}\right)$, controls the thermal evolution of magnetic domain states in grains as they cool below the Curie temperature, become blocked in local energy minima states, and acquire a thermoremanent magnetization. Equally important is the stability of domain states to subsequent chemical, thermoviscous or deformation (stress) induced remagnetization [e.g., Dunlop, 1990; Halgedahl, 1993]. In either case, the nucleation of new domains, the denucleation of preexisting domains, and the unpinning of domain walls from crystal defects are critically dependent on the interplay among exchange, magnetoelastic, magnetocrystalline, magnetostatic and Zeeman contributions that drive the micromagnetic spin structure of grains to minimum energy states. For example, the competing roles of magnetocrystalline, magnetoelastic and stress induced anisotropies affect the thermal dependence of coercivity resulting from the interactions between domain walls and crystal defects [Moskowitz, 1993a]. The magnitude and thermal behavior of these various energy contributions are determined by a particular material constant, such as $K_{1}$ and $\lambda$. Yet, experimental measurements of these fundamental magnetic constants at high temperatures

\section{Copyright 1995 by the American Geophysical Union.}

are poorly known for most magnetic minerals, including magnetite $\left(\mathrm{Fe}_{3} \mathrm{O}_{4}\right)$ and titanomagnetite $\left(\mathrm{Fe}_{3-\mathrm{x}} \mathrm{Ti}_{\mathrm{x}} \mathrm{O}_{4}, 0 \leq \mathrm{x} \leq 1\right)$.

Most previous studies on magnetostriction and magnetocrystalline anisotropy in titanomagnetites (TM) have focused on their thermal behavior below $300 \mathrm{~K}$ [Syono, 1965; Klerk et al., 1977; Kakol et al., 1991]. High temperature measurements have been limited to magnetite or Ti-poor TM $(x<0.1)$ [Klapel and Shive, 1974; Fletcher and O'Reilly, 1974]. For these compositions, magnetocrystalline anisotropy decreases rapidly with temperature, approximately as $\mathbf{M}_{\mathrm{s}}^{8}(\mathrm{~T})$. Özdemir and Moskowitz [1992] and Moskowitz [1993b] measured magnetostriction at elevated temperatures for polycrystalline samples of magnetite and Ti-rich TM. They found that the polycrystalline magnetostriction constant $\lambda_{s}(T)$ varied approximately as $M_{a}{ }^{n}(T)$ with $n=2-4$, depending on compositon.

Titanium-rich TM is an important magnetic mineral in ocean basalts, yet no measurements of single crystal magnetostriction constants or magnetocrystalline anisotropy between room temperature and $T_{c}$ exists for these phases. Recently it has been suggested that high ratios of saturation remanent magnetization to saturation magnetization $\left(M_{R} M_{s}\right)$ observed in fresh ocean basalts from the East Pacific Rise is due to high cubic magnetocrystalline anisotropy of TM [Kent and Gee, 1994]. However, high cubic anisotropy in TM is contrary to previous measurements of low values of $K_{1}$ for this phase [Syono, 1965; Kakol et al., 1991]. In this paper, high-temperature magnetocrystalline anisotropy and magnetostriction measurements are reported for a synthetic single crystal of $\mathrm{Fe}_{2.4} \mathrm{Ti}_{0.61} \mathrm{O}_{4}$ (TM61).

\section{Theory}

Magnetocrystalline anisotropy results from the coupling of the spin ionic moments to the crystalline lattice, producing crystallographic easy axes of magnetization. Magnetostriction is the fractional change in length of a magnetic crystal that accompanies the process of magnetization and produces a strain dependence of the anisotropy energy [e.g., Kittel, 1949; Kakol et al., 1991; Ye et al., 1994]. The total anisotropy energy will therefore include a term dependent on the crystallographic direction of magnetization and another dependent on strain. For cubic crystals like TM60, the anisotropy energy, neglecting second-order terms, is given by

$$
E_{a}=\left(K_{1}+K_{\lambda}\right)\left(\alpha_{1}^{2} \alpha_{2}^{2}+\alpha_{2}^{2} \alpha_{3}^{2}+\alpha_{3}^{2} \alpha_{2}^{2}\right)
$$

and

$$
K_{\lambda}=9 / 4\left(c_{11}-c_{12}\right) \lambda_{100}^{2}-9 / 2 c_{44} \lambda_{111}^{2}
$$

where $\alpha_{i}$ is the direction cosine of the magnetization with respect to the cubic axes, $K_{1}$ is the magnetocrystalline anisotropy constant, $\lambda_{100}$ and $\lambda_{111}$ are the magnetostriction constants along [100] and [111], and $c_{11}, c_{12}, c_{44}$ are elastic 
moduli [Kittel, 1949]. Because both contributions to the anisotropy energy have the same angular dependence, the effect of magnetostriction is simply to replace the usual $\mathrm{K}_{1}$ with an effective anisotropy constant $K^{\prime}=K_{1}+K_{\lambda}$.

If anisotropy measurements are made at constant strain, then magnetostriction would have no effect on the anisotropy constant and $K^{\prime}=K_{1}$. Usually anisotropy is measured under constant stress (ambient pressure conditions) so that the sample deforms under the action of magnetoelastic forces [Kittel, 1949]. In our experiments, the anisotropy constant measured at constant stress by torque magnetometry is therefore the sum of $K_{1}$ and $K_{\lambda}$. The thermal dependence of $K_{1}(T)$ can be obtained by subtraction if $K_{\lambda}$ is determined independently by measuring the thermal dependence of $\lambda_{100}$, $\lambda_{111}$, and the elastic moduli. Previous measurements of anisotropy constants in single crystal TM by Syono [1965] and Kakol et al. [1991] measure $\mathbf{K}^{\prime}$ not $\mathbf{K}_{\mathbf{l}}$ [Ye et al., 1994]. Nevertheless, it is the total anisotropy constant $\mathbf{K}^{\prime}$ that is important and is the appropriate constant to use for micromagnetic modeling of magnetic properties and domain states.

\section{Experimental Techniques}

A large (5-9 $\mathrm{mm}$ wide and $5 \mathrm{~cm}$ long) single crystal of TM 60 was grown along the $<100\rangle$ direction by the floating zone technique using a halogen lamp image furnace [Wanamaker and Moskowitz, 1994]. Examination of the crystal using optical and scanning electron microscopy, electron microprobe, thermomagnetic analysis, and Laue back-reflection camera shows it to be single crystal, single phase, and chemically homogeneous except for a small gradient in Ti/Fe along the growth dimension due to the different melt-solid distribution coefficients of these species. The chemical gradient along the growth direction is $\approx 0.75 \mathrm{~mole} \% \mathrm{Ti} / \mathrm{cm}$ and is negligible for the small (1-2 mm thick) samples used in the experiments. Two disk shaped samples (sample $A$ and B), $4 \mathrm{~mm}$ in diameter and $1 \mathrm{~mm}$ thick were cut parallel to the (001) plane from the top part of the crystal and used for anisotropy and magnetostriction measurements. Chemical composition obtained from electron microprobe analysis for another piece near the top of the crystal is $x=0.61$ [Wanamaker and Moskowitz, 1994].

Anisotropy measurements were made using a Digital Measurement Systems torque magnetometer. A magnetic field of $0.5 \mathrm{~T}$ was applied parallel to the plane of a disk-shaped sample, and was 10 times greater than the field needed to

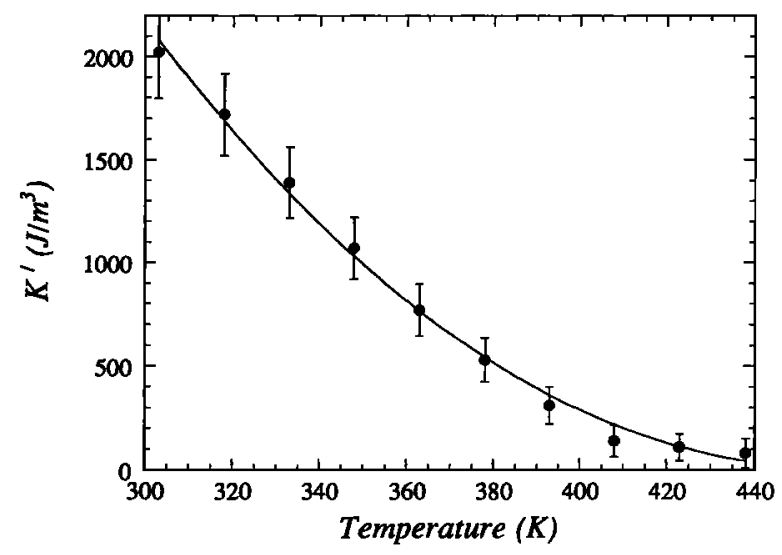

Figure 1. Anisotropy constant $\left(K^{\prime}\right)$ as a function of temperature. The solid line is power law fit to the equation $\left(\mathrm{T}_{\mathrm{c}} \mathrm{-T}\right)^{\mathrm{m}}$.

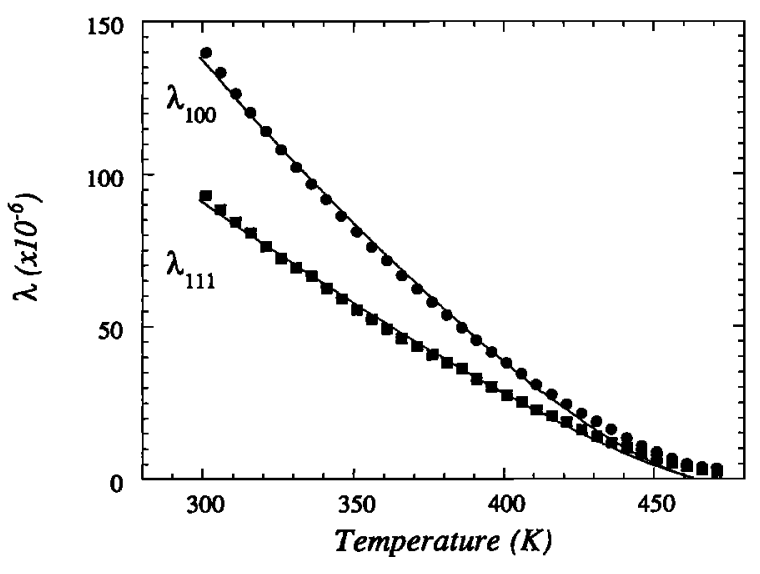

Figure 2. Magnetostriction constants $\lambda_{100}$ (solid circles) and $\lambda_{111}$ (solid squares) as a function of temperature. The solid lines are power law fits to the equation $\left(T_{c}-T\right)^{\mathbf{m}}$.

saturate the sample in the plane of the disk. For measurements in a (001) plane of a cubic crystal, the torque $L$ on the crystal per unit volume is

$$
L=-\frac{K^{\prime}}{2} \sin 4 \theta
$$

where $\theta$ is the angle between the magnetization $M$ and the easy axis [e.g., Cullity, 1972]. The anisotropy constant $K^{\prime}$ is obtained from Fourier analysis of the torque curves.

If there is no sample misalignment, then the torque curve varies as $\sin 4 \theta$. However, if the specimen, torque magnetometer, or both are slightly misaligned, then a spurious uniaxial $\sin 2 \theta$ component is produced. Besides misalignment errors, induced stress anisotropy will also produce a $\sin 2 \theta$ term. A small $\sin 2 \theta$ contribution was observed to be about $10 \%$ of the $\sin 4 \theta$ term and used an error measurement for $K^{\prime}$. Anisotropy measurements were made at 10 different temperatures between $303 \mathrm{~K}$ and $423 \mathrm{~K}$ in nitrogen gas.

Magnetostriction was measured with recording rotating field magnetostrictometer [Moskowitz, 1993b]. Metal strain gages (Micromeasurement type WK-06-031CF-350) were bonded parallel to the $\langle 100\rangle$ (sample $A$ ) and the $\langle 110\rangle$ (sample B) directions on the $\{100\}$ plane. Magnetostriction was measured at $1 \mathrm{~K}$ intervals between $300 \mathrm{~K}$ and $473 \mathrm{~K}$ as the sample was thermally cycled in nitrogen gas (Moskowitz, [1993b]). The maximum sensitivity for $\lambda$ was approximately $2 \times 10^{-8}$ and measurement uncertainty was $3 \%$.

The temperature dependence of magnetization was measured using a VSM in fields of $600,800,1000$ and 1200 $\mathrm{mT}$. $\mathrm{M}_{\mathrm{g}}(\mathrm{T})$ was determined as the intercept at $\mathrm{H}=0$ from linear fits of $M$ versus $H$ at constant temperature. The Curie temperature for sample $A$ is $465 \mathrm{~K}$ and indicates that it is slightly nonstoichiometric due to cation vacancies formed at high temperature during crystal growth [Wanamaker and Moskowitz, 1994]. In the temperature range studied (300-473K), $M_{2}$ was found to vary as $\left(T_{c}-T^{0.42}\right.$. The bulk coercivity $\left(\mathrm{H}_{\mathrm{c}}\right)$ at room temperature was $0.2 \mathrm{mT}$.

\section{Discussion}

At room temperature, $\mathrm{K}^{\prime}=2.02 \pm 0.22 \mathrm{~kJ} / \mathrm{m}^{3}$ and is positive, making the $<100\rangle$ directions the easy axes of magnetization in TM61. The magnitude of $\mathbf{K}^{\prime}$ is six times lower than the value for pure magnetite and agrees well with earlier studies on compositions close to TM60 [Syono, 1965; Kakol et al., 
1991]. A positive anisotropy constant for TM61 is also consistent with preliminary domain observations on the $\{110\}$ surface of a sample [Sahu and Moskowitz, 1993]. Near the center of the crystal, simple lamellar domains parallel to the $<100>$ easy direction were observed. Based on our anisotropy measurements, the intrinsic cubic-style domain patterns for TM61 should consist of arrays of $180^{\circ}$ walls with closure domains bounded by $90^{\circ}$ walls. In contrast, for magnetite and TMs with compositions less than $x \approx 0.55, K^{\prime}$ is negative at room temperature corresponding to $\langle 111\rangle$ easy axes [Fletcher and O'Reilly, 1974; Kakol et al., 1991] and $180^{\circ}$, $109^{\circ}$, and $71^{\circ}$ walls.

The thermal dependence of the magnetocrystalline anisotropy constant $\mathbf{K}^{\prime}$ is shown in Figure 1 . At elevated temperatures, $\mathbf{K}^{\prime}$ remains positive indicating that there is no isotropic point (ie., $K^{\prime} \neq 0$ or change sign) in TM61 up to the maximum measurement temperature of $423 \mathrm{~K}$ and probably extending to the Curie temperature $\left(T_{c}=465 \mathrm{~K}\right)$. Because at low temperatures $\mathbf{K}^{\prime}$ is large and positive for compositions with $x>0.5$ [Syono, 1965; Kakol et al., 1991], these results show that $\mathbf{K}^{\prime}$ is probably positive at all temperatures between absolute zero and $\mathrm{T}_{\mathrm{c}}$. Comparing our data with published low temperature $\mathrm{K}^{\prime}-\mathrm{T}$ results [Syono, 1965; Kakol et al., 1991] suggests that at some intermediate composition between TM55 and TM61, an isotropic point could exist within or below the blocking temperature range for TRM between $T_{c}$ and room temperature. This could lead to a rearrangement of domain structure as domain walls controlled by $\mathbf{K}^{\prime}$ become unpinned when $K^{\prime} \rightarrow 0$ and grains subsequently become remagnetized.

Figure 2 shows the thermal dependence of the magnetostriction constants for TM61. At $300 \mathrm{~K}, \lambda_{111}=95.4 \times 10^{-6}$ and $\lambda_{100}=142.5 \times 10^{-6}$ and are similar to values reported by Klerk et al. [1977] for single crystal TM60. A critical stress $\left(\sigma_{c}\right)$ for overriding the magnetocrystalline cubic anisotropy can be calculated from the experimental values of $\mathrm{K}^{\prime}, \lambda_{111}$, and $\lambda_{100}$ by equating the magnetocrystalline energy $\left(K^{\prime} / 4, K^{\prime}>0\right)$ with the magnetostriction energy $\left(1.5 \lambda_{2} \sigma_{c}\right)$, yielding $\sigma_{c}=3 \mathrm{MPa}$. Residual stresses at least 10 times larger than $\sigma_{c}$ have been estimated from uniaxial domain patterns typically observed in polycrystalline synthetic and natural Ti-rich TMs [Halgedahl, 1987; Moskowitz et al., 1988]. Our anisotropy measurements coupled with domain studies on TM confirm that intrinsic cubic anisotropy can be overcome easily by stress resulting in stress-induced uniaxial anisotropy.

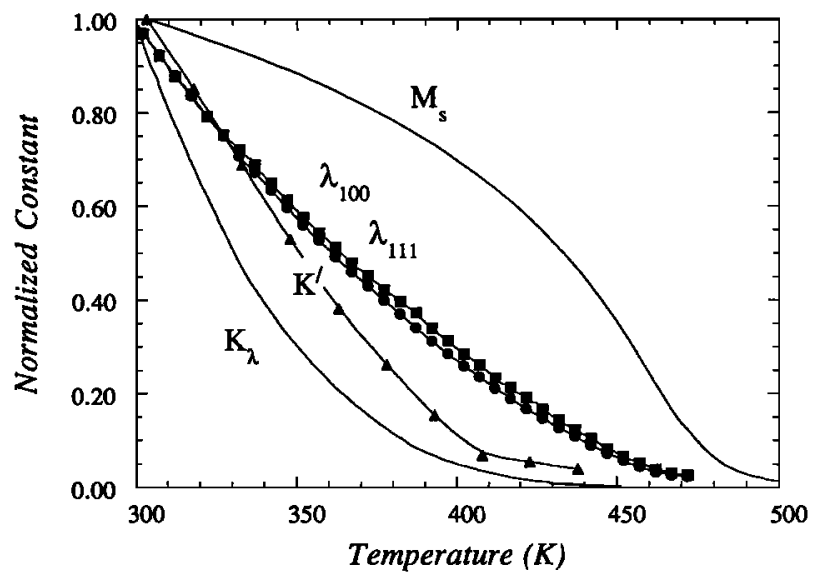

Figure 3. Normalized $M_{2}, \lambda_{100}$ (solid circles), $\lambda_{111}$ (solid squares), $K^{\prime}$ (solid triangles), and $K_{\lambda}$ for TM61 as a function of temperature. $K_{\lambda}$ is calculated from (2) with $c_{11}-c_{12}=$ $9.42 \times 10^{10} \mathrm{~N} / \mathrm{m}^{2}$ and $c_{44}=6.57 \times 10^{10} \mathrm{~N} / \mathrm{m}^{2}$.
The thermal dependence of the anisotropy and magnetostriction constants were fit to a power law equation in terms of the reduced temperature $\left(\mathrm{T}_{\mathrm{c}}-\mathrm{T}\right)^{\mathrm{m}}$ with $\mathrm{T}_{\mathrm{c}}=465 \mathrm{~K}$. Reasonable power law fits were obtained and are shown as solid lines in Figures 1 and 2. Over the entire temperature range, the index $\mathrm{m}$ is equal to $2.2 \pm 0.2,1.37 \pm 0.01,1.26 \pm 0.01$, and $1.31 \pm 0.01$ for $K^{\prime}, \lambda_{100}, \lambda_{111}, \lambda_{z}$, respectively. The thermal dependence of $\lambda_{\mathrm{s}}$ obtained from the single crystal measurements is nearly identical to results obtained on polycrystalline TM60 [Moskowitz, 1993b]. In terms of $\mathbf{M}_{\mathrm{z}}^{\mathrm{n}}$, the power law index $n$ is equal to $5.8 \pm 0.32,3.57 \pm 0.07,3.28 \pm 0.06$, and $3.42 \pm 0.07$ for $K^{\prime}, \lambda_{100}, \lambda_{111}, \lambda_{4}$, respectively.

The thermal dependence of the various constants are plotted in normalized form in Figure 3. $K^{\prime}$ shows a much weaker temperature dependence at elevated temperatures $(n \approx 6)$ than that observed for magnetite $(n=8-10)$ confirming that the magnetoelastic component (equ. (2)) rather than the magnetocrystalline component of $\mathbf{K}^{\prime}$ is the dominant term for TM61. $K^{\prime}(T)$ exhibits a thermal dependence intermediate between $\lambda^{\prime}(\mathrm{T})$ and $\lambda^{2}(\mathrm{~T})$ (Figure 3). A linear dependence on $\lambda(\mathrm{T})$ would be associated with a uniaxial stress anisotropy component resulting from residual stresses in the crystal.

The temperature dependence of $K_{1}$ alone cannot be obtained using just the torque measurements [Kittel, 1949; Ye et al., 1994]. In principle, $K_{1}(T)$ can be obtained by subtracting the magnetoelastic contribution from the total anisotropy constant, $K_{1}(T)=K^{\prime}(T)-K_{\lambda}(T)$, where $K_{\lambda}$ is calculated from equation (2) using the single crystal values of $\lambda_{111}$ and $\lambda_{100}$. However, the elastic constants for TM61 are not known directly and must be estimated by interpolation between room temperature measurements of $c_{11}, c_{12}$, and $c_{44}$ for TM0 and TM95 [Syono et al., 1971]. Moreover, the elastic properties of TM95 are anomalous with respect to Ti-poor TMs due to Jahn-Teller lattice distortion effects of tetrahedral $\mathrm{Fe}^{+2}$ [Syono et al., 1971], so a simple linear interpolation scheme between TM0 and TM95 is unreliable. To compensate for this effect, we follow the model of Kakol et al. [1991] which assumes that the elastic constants are directly proportional to the concentration of tetrahedral $\mathrm{Fe}^{2+}$ for $\mathrm{x}>0.2$. This assumes that (1) c's vary linearly between TM20 and TM95 (instead of TM0 and TM95); (2) $\mathrm{c}_{\mathrm{i}}$ 's are independent of composition for $x<0.2$ and equal to the values for TM0; and (3) $c_{i}$ 's are independent of temperature. Following this procedure we obtain the curve marked $K_{1}$ (cal) in Figure 4.

At room temperature $K_{1}$ is positive and less than $25 \%$ of $K^{\prime}$. The calculated $K_{1}$ thermal dependence is unexpected considering that standard theory predicts that $K_{1}$ varies as a high

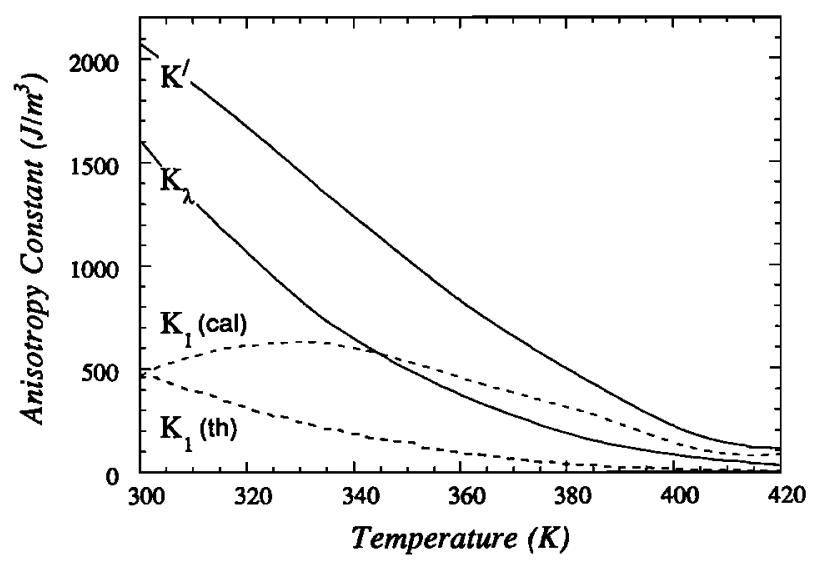

Figure 4. Anisotropy constants $K^{\prime}, K_{\lambda}, K_{1}$ as a function of temperature. $\mathbf{K}^{\prime}$ is experimentally determined, $K_{\lambda}$ is calculated from (2), $K_{1}($ cal $)=K^{\prime}-K_{\lambda}$, and $K_{1}($ th $) \propto M_{a}{ }^{10}$. 
power of the magnetization [Callen and Callen, 1963]. In cubic crystals, the theoretical relationship is $\mathrm{K}_{1}-\mathbf{M}_{\mathrm{a}}{ }^{10}$, which is shown in Figure 4 as the curve marked $K_{1}(t h)$. The poorly constrained elastic constants or an unaccounted for stress anisotropy term may contribute to a potentially spurious $\mathbf{K}_{\mathbf{1}}$ variation with temperature. The absolute magnitudes of the elastic constants are important because both $K_{1}$ and $K_{\lambda}$ are calculated as the difference between two terms. Data presented by Syono et al. [1971] on the temperature dependence of $c_{11}-c_{12}$ and $c_{44}$ in the paramagnetic region between $T_{c}$ and $300 \mathrm{~K}$ for TM95 show that $\mathrm{c}_{11}-\mathrm{c}_{12}$ increases whereas $\mathrm{c}_{44}$ decreases with increasing temperature. This type of thermal dependence, if applied to TM61, would result in larger values of $K_{\lambda}$, smaller or even negative calculated values of $K_{1}$, and a $K_{1}(T)$ behavior different from the one shown in Figure 4. Ferrimagnets with two sublattice magnetizations can show complex $K_{1}-T$ behavior based on the Neel-Wolf crystal field model of one-ion anisotropy [Wolf, 1957], but usually this type of behavior is observed below $300 \mathrm{~K}$ (e.g., the Verwey transition in magnetite). Regardless of the exact $K_{1}$ dependence, the relevant parameter for micromagnetic models and domain studies is $\mathbf{K}^{\prime}$, which was measured directly in the present study and well constrained.

\section{Conclusions}

1. The magnetocrystalline anisotropy constant $\mathrm{K}^{\prime}$ at $23^{\circ} \mathrm{C}$ for TM61 is $2.02 \mathrm{~kJ} / \mathrm{m}^{3}$. $\mathrm{K}^{\prime}$ has a positive sign making the $<100>$ crystal directions the easy axes of magnetization. The low value of $\mathbf{K}^{\prime}$ agrees well with earlier studies on compositions close to TM60 [Syono, 1965; Kakol et al., 1991].

2. Above room temperature, $K^{\prime}$ remains positive and varies as (1-T/T $\left.)_{0}\right)^{2.2}$ and $M_{a}(T)^{6}$ and exhibiting a thermal dependence intermediate between $\lambda^{1}(T)$ and $\lambda^{2}(T)$. $K^{\prime}$ shows a weaker temperature dependence at elevated temperatures $(n=6)$ than that observed for magnetite $(n=8-10)$ confirming that the magnetoelastic rather than the magnetocrystalline component of $\mathbf{K}^{\prime}$ is dominant for TM61.

3. $\mathbf{K}^{\prime}$ is low at room temperature for TM61 suggesting that cubic anisotropy plays a secondary role relative to stress or shape anisotropy in controlling magnetic behavior in rocks containing intermediate TM, such as fresh and altered oceanic basalts.

Acknowledgments. We thank M. Hintz (3M Company, St. Paul, MN) for the anisotropy measurements. The authors gratefully acknowledge reviews by S. Halgedhal and Ö. Özdemir. This research was supported by NSF Grant EAR-9017389 and is contribution 9413 of the Institute for Rock Magnetism. The IRM is funded by grants from the Keck and National Science Foundations and the University of Minnesota.

\section{References}

Callen, E. and H. Callen, Static magnetoelastic coupling in cubic crystals, Phys. Rev., 129, 578 -593, 1963.

Cullity, B. D., Introduction to Magnetic Materials, 666pp., Addison-Wesley, Reading, Mass., 1972.
Dunlop, D. J., Developments in rock magnetism, Rep. Prog. Phys., 53, 707-792, 1990.

Fletcher, E., and W. O'Reilly, Contribution of $\mathrm{Fe}^{2+}$ ions to the magnetocrystalline anisotropy constant $\mathrm{K}_{1}$ of $\mathrm{Fe}_{3-x} \mathrm{Ti}_{2} \mathrm{O}_{4}$ (0<x<0.1), J. Phys., C7, 171-178, 1974.

Halgedahl, S. L., Domain pattern observations in rock magnetism: progress and problems, Phys. Earth Planet. Inter., 46, 127-163, 1987.

Halgedahl, S.L., Magnetic domain patterns observed on synthetic Ti-rich titanomagnetite as a function of temperature and in states of thermoremanent magnetization, J. Geophys. Res., 96 , 3943-3972, 1993.

Kakol, Z., J. Sabol and J. M. Honig, Magnetic anisotropy of titanomagnetites $\mathrm{Fe}_{3-\mathrm{x}} \mathrm{Ti}_{\mathrm{z}} \mathrm{O}_{4}, 0<\mathrm{x}<0.55$, Phys. Rev. B, 44, 2198-2204, 1991.

Kent D.V., and J. Gee, Grain size-dependent alteration and the magnetization of oceanic basalts, Science, 265, 1561-1563, 1994.

Kittel, C., 1949, Physical theory of ferromagnetic domains, Rev. Mod. Phys., 21, 541-583, 1949.

Klapel, G., and P. Shive, High temperature magnetostriction of magnetite, J. Geophys. Res., 79, 2629-2633, 1974.

Klerk, J., V. A. M. Brabers, and A. J. M. Kuipers, Magnetostriction of the mixed series $\mathrm{Fe}_{3-2} \mathrm{TixO4}$, J.Phys., 38, C1, 187-189, 1977.

Moskowitz, B.M., Micromagnetic study of the influence of crystal defects on coercivity in magnetite, J. Geophys. Res., 98, 18,011-18,026, 1993a.

Moskowitz, B. M., High-temperature magnetostriction of magnetite and titanomagnetite, J.Geophys.Res., 98, 359-371, $1993 b$.

Moskowitz, B.M., S.L. Halgedahl, and C.A. Lawson, Magnetic domains on unpolished and polished surfaces of titanium-rich titanomagnetite, J. Geophys. Res., 93, 3372-3386, 1988.

Özdemir, Ô., and B. M. Moskowitz, Magnetostriction in aluminum-substituted titanomagnetites, Geophys. Res. Lett., 19, 2361-2364, 1992.

Sahu, S., and B. M. Moskowitz, Domain patterns on single crystal titanomagnetite (TM60) using the Bitte: method and magneto-optic Kerr effect (MOKE) (abstract), Eos Trans. $A G U, 74,203,1993$.

Syono, $Y$., Magnetocrystalline anisotropy and magnetostriction of $\mathrm{Fe}_{3} \mathrm{O}_{4}-\mathrm{Fe}_{2} \mathrm{TiO}_{4}$ series, with special application to rock magnetism, Jpn. J. Geophys., 4, 71-143, 1965.

Syono, Y., Y. Fukai, and Y. Ishikawa, Anomalous elastic properties of $\mathrm{Fe}_{2} \mathrm{TiO}_{4}$, J. Phys. Soc. Jpn., 31, 471-476, 1971.

Wanamaker, B.J., and B. M. Moskowitz, Effect of nonstoichiometry on the magnetic and electrical properties of synthetic single crystal $\mathrm{Fe}_{2.4} \mathrm{Ti}_{0.6} \mathrm{O}_{4}$, Geophys. Res. Lett., 21 983-986, 1994.

Wolf, W.P., Effect of crystalline electric fields on ferromagnetic anisotropy, Phys. Rev., 108, 1152-1157, 1957.

Ye, J., A.J. Newell, and R.T. Merrill, A re-evaluation of magnetocrystalline anisotropy and magnetostriction constants, Geophys. Res. Lett., 21, 25-28, 1994.

B. Moskowitz and S. Sahu, Dept. of Geology and Geophysics, 310 Pillsbury Dr., SE, University of Minnesota, Minneapolis, MN 55455 (e-mail: bmosk@maroon.tc.umn.edu; sahu0001@gold.tc.umn.edu)

(Received September 29, 1994; accepted November 4, 1994) 